\title{
How Age and Disability Affect Long-Term Care Expenditures in the United States
}

\author{
Sally C. Stearns*, Edward C. Norton* and Zhou Yang** \\ * University of North Carolina at Chapel Hill, North Carolina \\ **University of Florida, Gainesville, Florida \\ E-mail: Sally_Stearns@unc.edu
}

The ageing of the population and increasing longevity result in predictions of sizeable increases in long-term care expenditures. Other analyses have shown significant decreases in disability prevalence among older people in the US. This study provides an empirical quantification of the net result of these two forces (increased expenditures due to ageing versus potential expenditure reductions due to reduced disability) using the Medicare Current Beneficiary Survey. The analyses show that the implications of ageing and increasing longevity for long-term care expenditures are modest relative to the effects of future increases or decreases in functional abilities of older people.

\section{Introduction}

The ageing of the population combined with projections of increasing longevity result in predictions of substantial increases in future long-term care expenditures. Both acute and long-term care health expenditures increase with age primarily for two reasons: (1) chronic disease incidence and prevalence increase with age, and (2) mortality increases with age. The implications of increasing longevity for health care expenditures, especially long-term care expenditures, therefore depend critically on the competing theories of compression or expansion of morbidity (Fries, 1980; Olshansky and Ault, 1986). As people live longer, lifetime health expenditures will increase, but the amount of increase will be determined by whether the onset of chronic disease and disability is being delayed at a rate greater than, less than, or equal to the changing rate of disability.

Manton and Gu (2001) found decreases in the age-adjusted prevalence of chronic disability among older individuals in the United States using National Long-Term Care Survey data from 1982-1999, with the rate of decline increasing from 0.26 per cent per year in the 1980s to a decline of 0.56 per cent per year. A recent reanalysis of these data found, however, that most of the improvements were among people with less severe disability (Spillman, 2004) Getting comparable estimates for countries other than the US is complicated by the lack of longitudinal data and by varying definitions of disability in the available data sets. National household survey evidence from a number of developed countries suggests that the proportion of the extra years of life spent in disabled states rose between 1970 and 1990, but less time was spent in the most disabled states (Bone et al., 1995; Cambois and Robine, 1996; Robine et al., 1997; OECD, 1998; Bone et al., 1998; Bebbington and Comas-Herrera, 2000; Bissett, 2002). Therefore, unlike the US, in many developed countries healthy life expectancy may not be rising as quickly as life expectancy, although the proportion of time spent in the most disabled states (which are potentially the most health service use intensive) appears to be declining. 
While disability trends are undoubtedly the driving force in long-term care expenditures, a more subtle concern pertains to the competing roles of age and increasing longevity. A substantial literature documents that proximity to death - rather than age per se - plays a pivotal role in acute health care expenditure predictions (Zweifel et al., 1999; Spillman and Lubitz, 2000; Yang et al., 2003; Stearns and Norton, 2004; Seshamani and Gray, 2004). A smaller body of evidence shows that disease status (Seshamani and Gray, 2004) or functional ability (Cutler and Meara, 2001a and 2001b) may modify the relationship between age, proximity to death, and health expenditures. In particular, Cutler and Meara (2001b) show that the effects of disability and time to death on health expenditures explain most of the age effect and are largely independent. Only a limited literature has looked at long-term care expenditures in relation to proximity to death (Spillman and Lubitz, 2000; Spillman and Lubitz, 2002; McGrail et al., 2000; Yang et al., 2003).

This study explores how two factors - age and functional status - affect long-term care expenditures, and thus gains insights into the implications of increasing longevity and changing disability. While long-term care expenditures will increase with both age and disability, the relative effects are important to understand in order to gauge the increases in expenditures that could occur from increasing longevity relative to any offsets in expenditures from improvements in functional status. Even if disability rates are stable, the estimation provides insights into the implications of increasing longevity for long-term care expenditures.

Models of long-term care expenditures are estimated for older Medicare beneficiaries in the United States using data from the Medicare Current Beneficiary Survey (19921998). The marginal effects of increasing age and increasing functional impairment are calculated and compared. Implications of the differences in estimated effects are considered in terms of their relative differences as well as implications for recent simulations of the effects of changing disability on long-term care expenditures.

\section{Conceptual model and methods}

Long-term care expenditures for an individual are a function of a number of factors, including socio-demographic characteristics (age, gender, race/ethnicity, education, marital status, family structure including number of children and proximity to grown children), economic circumstances (income, insurance, wealth), health status including disease presence and functional ability (measured here by activities of daily living (ADLs) and instrumental activities of daily living (IADLs)), and geographic factors (including provider supply and regional utilization patterns) (Norton, 2000). Rather than estimate a fully-specified model, however, we use a simplified model that focuses on the effects of age and ADL impairment while controlling for a limited number of additional factors:

$$
\begin{aligned}
L T C_{i t}= & \beta_{0}+\beta_{1} A G E_{i t}+\beta_{2} A D L_{i t}+\beta_{3} A G E_{i t} \times A D L_{i t}+\beta_{4} T T D_{i t}+\beta_{5} A G E_{i t} \times T T D_{i t} \\
& +\beta_{5} I A D L O N L Y+\beta_{6} S O C I O D E M O_{i t}+\beta_{7} G E O_{i t}+\beta_{8} \text { TIMEDUMMIES }_{i t}+\varepsilon_{i t}
\end{aligned}
$$

The model shown in Equation 1 includes age and ADL interactions to allow for varying effects of functional status on long-term care expenditures at different ages. Measures of time to death (TTD) are included because of the substantial evidence cited earlier on the importance of controlling for proximity to death in models of health expenditures. If 
longevity is constant, the failure to control for proximity to death is not a problem per se (as predictions done by age will be unbiased on average). If longevity is increasing (as it has been in the United States), however, then predictions from models that account for the age distribution but not the role of proximity to death will be biased (Stearns and Norton, 2004). The interaction of age and time to death is included to account for the possibility that end-of-life expenditures change at different ages (Spillman and Lubitz, 2000; Yang et al., 2003). We do not include interactions of disability and time to death because of the evidence that the effects of these two factors are largely independent (Cutler and Meara, 2001a).

The model includes indicators of sociodemographics (gender, race, marital status), geographic region (census region and rural indicator), and month and year dummy variables. While a myriad of other factors such as education and number of children will affect long-term care expenditures, we exclude them from the estimated models to focus on the underlying effects of age and ADL limitations, and the fact that data on these measures (age distribution, ADLs, and proximity to death) are often available when more detailed measures are not available. Furthermore, some factors such as marital status may be jointly determined by age and health/functional status, which means they are 'endogenous' in the sense they are also functions of age, health status, and other unobserved variables. Inclusion of endogenous variables can lead to biased estimates of regression coefficients, so additional estimations were conducted to test the effect of controlling for marital status. Since these analyses did not result in substantially different results, marital status is included in the final estimations.

Because many people may not have any health care expenditures in a given time period (e.g., within a month), we estimate a two-part model (Diehr et al., 1999). Expenditures equal the likelihood of any long-term care expenditures multiplied by the level of expenditures conditional on having some expenditure during the time period for all our model estimations

$$
L T C_{i t}=\operatorname{Pr}(A n y L T C \mid X)_{i t} \times(\text { Level of LTC Expenditures } \mid X, \text { Any LTC })_{i t}
$$

where $X$ includes all independent variables defined above.

We use a logit model to estimate the first part of Equation (2) (the likelihood of use) because the dependent variable is a dichotomous measure of whether the person had any long-term care expenditures in a month. We use ordinary least squares (OLS) to estimate the second part of Equation (2) (the level of use conditional upon some use) because the dependent variable is a continuous measure. Because the error term for variables like health expenditures has a skewed rather than a normal distribution, we use logged expenditures as the dependent variable for the OLS estimation (Manning and Mullahy, 2001). Our interest, however, is not in predicted log expenditures, so we predict unlogged expenditures from a regression in which the dependent variable is logged, and correct for the fact that the error term does not have a normal distribution using the wellknown smearing estimator (Duan, 1983). We use the survey weights to obtain estimates of population parameters because the data set has a complex survey design (Little, 2004).

We determine the marginal effects of changes in age and ADL impairment by first predicting long-term care expenditures for the full sample. Then we simulate, separately, the marginal effect of increasing age by one year and the marginal effect of increasing the total number of ADL impairments by one on predicted long-term care expenditures. 
The simulations use parameters from both parts of the two-part model (e.g., Van Houtven and Norton, 2004). Because of the two-part model, we cannot easily obtain the analytical standard deviations of the marginal effect of age or total number of ADLs on long-term care expenditures. Instead, we use bootstrapping with repeated sampling to get 95 per cent confidence intervals for the estimated marginal effects for increasing age and increasing disability (Efron and Tibshirani, 1986; Ai and Norton, 2000). Because we use bootstrapping to get the confidence intervals for the estimates, we do not need to adjust the standard errors of the regression coefficients for clustering due to the complex survey design.

To highlight the different effects of increasing age and increasing disability, we graph the marginal effects in two ways: by age category, and by total number of ADL impairments. This comparison allows us to understand the relative contributions of increasing age and increasing (versus stable) ADL impairment according to age and disability groupings.

\section{Data}

The analysis uses data from the Medicare Current Beneficiary Survey (MCBS) Cost and Use Files for years 1992 to 1998 . The MCBS is a nationally representative longitudinal survey of all Medicare beneficiaries in the United States, including community-based individuals as well as persons living in institutions (Adler, 1994). The MCBS has several characteristics that make it ideal for research on longevity and health care expenditures. First, the sample is large, with roughly 12000 respondents per year. Second, the survey uses a rotating panel design, and respondents are followed for up to three years after the baseline interview unless they die or are lost to follow-up. Third, the cost and use files contain information on both decedents and survivors.

Our analysis includes MCBS respondents age 66 and older who were followed for more than one year, because our goal was to estimate the effect of changes in age and functional status. Because we are interested in long-term care expenditures as people age, we created an analysis file at the person-month level. Medicare expenditures were assigned to months according to the date of service, and all expenditures were inflated to 1998 dollars (1998\$) using the medical care component of the Consumer Price Index. We defined long-term care expenditures as the sum of all nursing home and home health expenditures by any payer (out-of-pocket, Medicaid, Medicare or other). If no long-term care expenditures occurred in a month, the level of expenditures was coded as zero.

Including survivors (i.e. people who did not die within the observation period) in the sample created a potential bias because we could not determine when the survivors died following their last interview. To minimize this potential bias, we used dummy variables representing number of months until censoring (i.e. to the last observation) to control for the fact that we did not know which individuals would die during the year following their last survey. In addition, we interacted the dummies for time until censoring with age (i.e. analogous to the variables interacting age with time to death) to treat survivors and decedents similarly.

As shown in Table 1, the final analysis file has 735339 observations at the personmonth level, representing 25403 unique people. 30 per cent of the sample (7751 people) had long-term care expenditures in at least one month. 17 per cent of the sample died during the survey follow-up period. People were followed on average for 29 months. Over the whole sample, long-term care expenditures averaged \$324 per month. Long-term care 
Table 1 Sample statistics: long-term care expenditures and age and ADL distributions (sample statistics)

\begin{tabular}{lcc}
\hline \hline & Total sample & Months with LTC expenditures \\
\hline Number of person-month observations & 735,339 & 87,741 \\
Number of people & 25,403 & 7,751 \\
Long-term care expenditures (1998 US\$) & & \\
Nursing home expenditures per month & $\$ 278$ & $\$ 2,329$ \\
Home health expenditures per month & $\$ 46$ & $\$ 390$ \\
Total LTC expenditures per month & $\$ 324$ & $\$ 2,719$ \\
Age distribution (percent of sample) & & \\
$66-70$ & $26.3 \%$ & $10.1 \%$ \\
$71-75$ & $20.9 \%$ & $9.3 \%$ \\
$76-80$ & $21.3 \%$ & $17.6 \%$ \\
$81-85$ & $18.2 \%$ & $27.5 \%$ \\
86-90 & $9.7 \%$ & $22.9 \%$ \\
91 and older & $3.6 \%$ & $12.6 \%$ \\
ADL limitations (percent of sample) & & \\
0 ADLs requiring assistance & $64.1 \%$ & $25.8 \%$ \\
1 ADLs requiring assistance & $12.7 \%$ & $7.1 \%$ \\
2 ADLs requiring assistance & $7.0 \%$ & $8.1 \%$ \\
3 ADLs requiring assistance & $4.1 \%$ & $8.0 \%$ \\
4 ADLs requiring assistance & $3.4 \%$ & $8.3 \%$ \\
5 ADLs requiring assistance & $4.3 \%$ & $17.4 \%$ \\
6 ADLs requiring assistance & $4.4 \%$ & $25.3 \%$ \\
\hline \hline
\end{tabular}

expenditures averaged $\$ 2719$ in months with positive long-term care expenditures, of which nursing home expenditures accounted for $\$ 2329$, while home health expenditures accounted for $\$ 390$. The age and ADL distributions of the sample-month observations show that long-term care expenditures occur relatively more frequently at older ages and among people with higher levels of functional limitations.

While the MCBS is a very rich longitudinal survey in many ways, it is important to note that $\mathrm{ADL} / \mathrm{IADL}$ functioning is only measured at one point each year. The relatively large sample size of the MCBS helps ensure that the estimates reflect the relationship, on average, between a point-in-time measure of disability and long-term care expenditures. Within-person variance in ADL measurement (both within a year and over a longer period of time), however, may correlate to long-term care expenditures in important ways that are not reflected in the analysis.

\section{Results}

The results from the two-part regression models show that most of the variables, or sets of variables, specified in Equation (1) are statistically significant. ${ }^{1}$ Because of the large number of interactions, the results are best understood by calculating predicted expenditures and then considering the marginal effects of a one-year increase in age or the onset of an additional ADL limitation. Table 2 provides the estimated mean change in longterm care expenditures from these changes according to two different groupings: by fiveyear age groups, and by total ADL limitations. Figures 1 and 2 provide the bootstrapped 
Table 2 Estimated marginal effects of increasing age and ADL impairment on long-term care expenditures (in US\$ per month)

\begin{tabular}{lcc}
\hline \hline $\begin{array}{l}\text { Marginal effect on long-term care } \\
\text { expenditures of: }\end{array}$ & $\begin{array}{l}\text { A one-year } \\
\text { increase in age }\end{array}$ & $\begin{array}{l}\text { An additional ADL } \\
\text { impairment }\end{array}$ \\
\hline Age distribution & $\$ 0$ & \\
$66-70$ & $\$ 12$ & $\$ 36$ \\
$71-75$ & $\$ 19$ & $\$ 110$ \\
$76-80$ & $\$ 23$ & $\$ 214$ \\
$81-85$ & $\$ 42$ & $\$ 381$ \\
$86-90$ & $\$ 9$ & $\$ 566$ \\
91 and older & $\$ 12$ & \\
ADL limitations & $\$ 7$ & $\$ 136$ \\
0 ADL requiring assistance & $\$ 20$ & $\$ 79$ \\
1 ADLs requiring assistance (or 0 to 1$)$ & $\$ 26$ & $\$ 647$ \\
2 ADLs requiring assistance (or 1 to 2) & $\$ 36$ & $\$ 120$ \\
3 ADLs requiring assistance (or 2 to 3) & $\$ 37$ & $\$ 957$ \\
4 ADLs requiring assistance (or 3 to 4) & $\$ 36$ & \\
5 ADLs requiring assistance (or 4 to 5) & & \\
6 ADLs requiring assistance (or 5 to 6) & & \\
\hline \hline
\end{tabular}

Note: Estimates adjusted for survey weights.

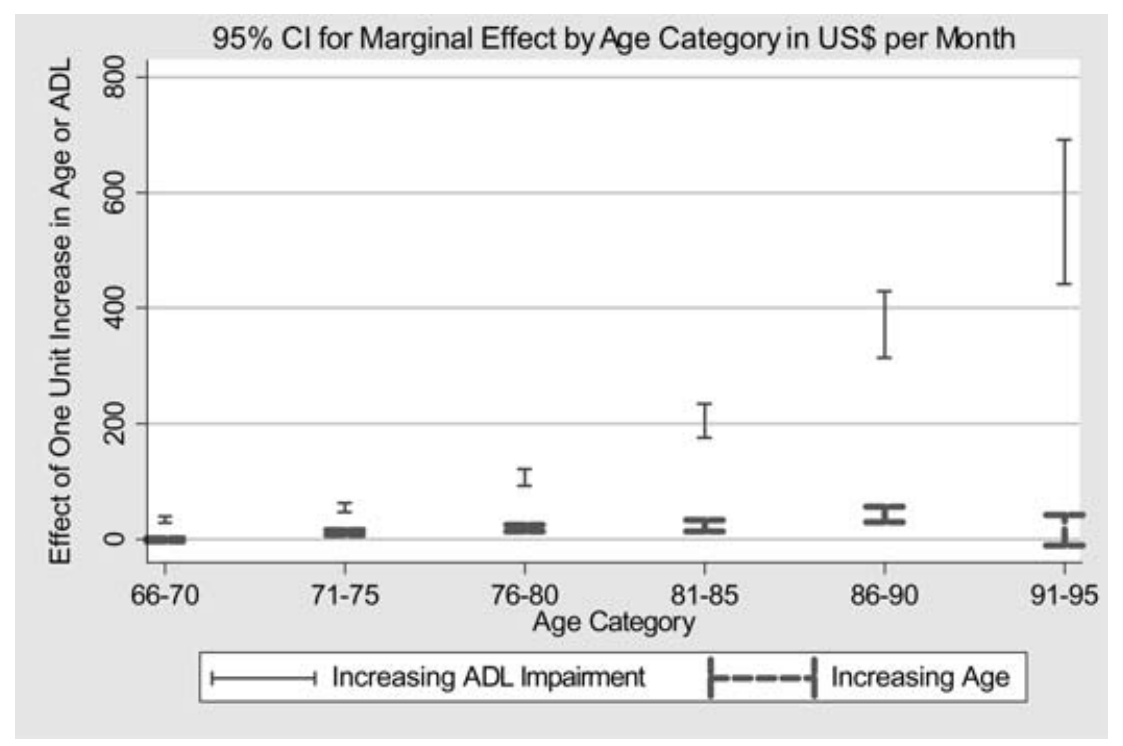

Figure 1. Impact of Age or ADLs on LTC Expenditures.

95 per cent confidence intervals $(\mathrm{Cls})$ for these two different groupings of the estimates of the marginal effects on long-term care expenditures. Assessing the marginal effects by both age and ADL grouping allows insights in the relative effects of changing longevity and disability status. 


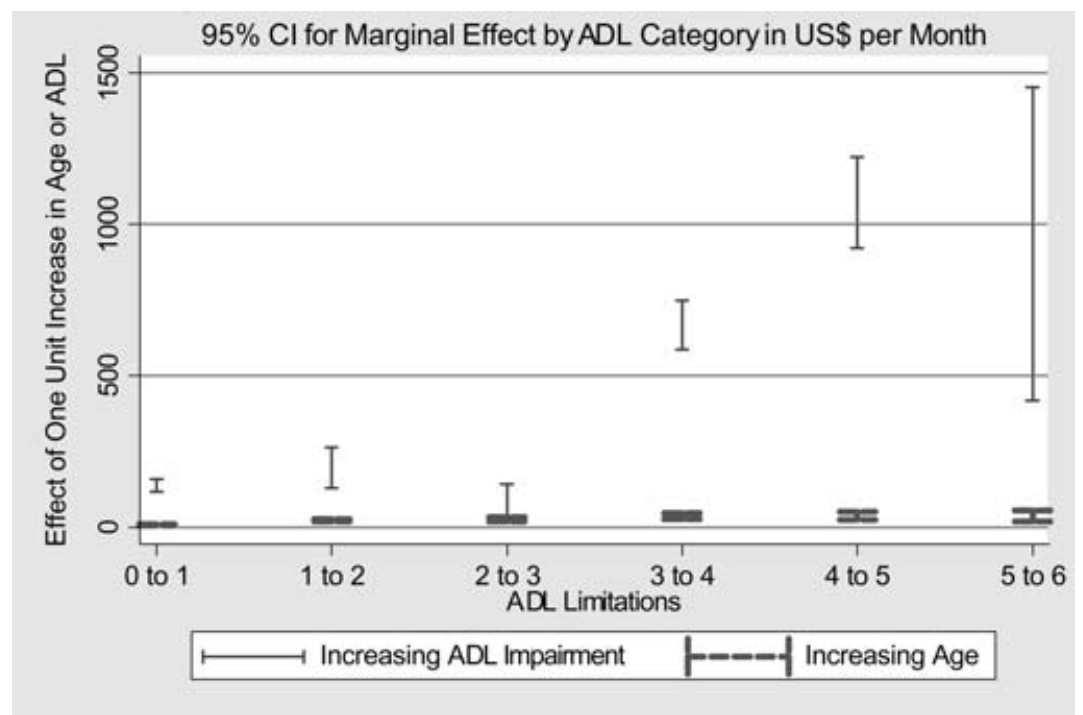

Figure 2. Impact of Age or ADLs on LTC Expenditures.

The patterns, which are interesting although not unexpected, are best seen in the figures. Increasing age (or longevity) results in an increase in long-term care (LTC) expenditures for some of the age groups, as shown in the bottom set of confidence intervals in Figure 1. The effect of a one-year increase in age is never greater than $\$ 42$ (on average per month) for persons aged 86-90, and increases in age do not significantly increase long-term care expenditures for the young elderly (66-70 years old) or the oldest elderly studied (91-95 years old). In contrast, impairment in one additional ADL category is predicted to lead to a substantially greater increase in long-term care expenditures. For example, among people aged 86-90, an increase in total ADL limitations by one for everyone in that age group would result in an increase of $\$ 381$ per month on average in long-term care expenditures. Because the age group reflects a five-year age span, the maximum expected increase from a full five-year increase in age (longevity) would be $\$ 210$ per month ( $=\$ 42 \times 5)$, which is less than two-thirds of the increase in expenditures from a one unit increase in ADL impairments. The magnitude of the effect of increasing disability is even greater for persons over age 90, especially in relation to the nonsignificant effect of increasing age (controlling for disability and time to death for this group).

Figure 2 uses the same predictions of long-term care expenditures but groups the predictions according to ADL limitations rather than age group. The marginal effect of an increase in age (or longevity) of one year is significantly greater than zero but modest for most of the ADL groups, with the highest mean value at $\$ 37$ for persons with 5 ADLs. More striking is the effect of an increase in ADL impairment, both in terms of the pattern of the effects and the magnitude for some of the groups. First, the effect of an increase in $\mathrm{ADL}$ limitations by one is relatively modest for up to $3 \mathrm{ADL}$ impairments, ranging from an estimated (mean) increase in monthly long-term care expenditures of $\$ 79$ (moving from 2 to 3 ADL impairments) to $\$ 202$ (moving from 1 to 2 ADL impairments). The increase 
Table 3 Life expectancy by age group and gender, in years: 1970-1997

\begin{tabular}{lcccc}
\hline \hline & 1970 & 1980 & 1990 & 1997 \\
\hline At age 65 & & & & \\
$\quad$ Total & 15.0 & 16.5 & 17.3 & 17.7 \\
Men & 13.0 & 14.2 & 15.1 & 15.9 \\
$\quad$ Women & 16.8 & 18.4 & 19.0 & 19.2 \\
At age 85 & & & & \\
$\quad$ Total & 5.3 & 6.0 & 6.2 & 6.3 \\
Men & 4.7 & 5.1 & 5.3 & 5.5 \\
Women & 5.6 & 6.4 & 6.7 & 6.6 \\
\hline \hline
\end{tabular}

Source: National Vital Statistics System.

http://www.efmoody.com/estate/lifeexpectancy.html (March 21, 2006)

in long-term care expenditures from additional impairments beyond 3 ADLs, however, is substantial, and is estimated to be as high as an additional $\$ 1,120$ per month for persons moving from 4 to $5 \mathrm{ADL}$ impairments. Indeed, the marginal effect of increasing ADL impairments decreases beyond this point, though the wide confidence interval for the estimate of the effect of moving from 5 to $6 \mathrm{ADL}$ impairments most likely is due to both a small sample size for this subgroup and greater variability in needs for person progressing to this level of impairment.

\section{Discussion}

The estimations in the preceding section provide further evidence that increases in longevity alone are not likely to result in substantial increases in long-term care expenditures. While the ageing of the population will undoubtedly increase the absolute level of long-term care expenditures, increases in per capita expenditures for this population due to ageing alone are likely to be limited. The analyses highlight, however, the critical role of disability and functional status in constraining or expanding longterm care expenditures. If age-specific disability levels either decrease or at least are held constant, the increase in long-term care expenditures may be contained. If, however, age-specific disability levels (ADL impairments) increase, then increasing longevity will result in increased disability and substantial increases in long-term care expenditures, especially when the increase in impairments exceeds three or more ADL difficulties.

While the calculations in the preceding section were made for increasing ADL impairment, the evidence within the United States over the past two decades has been toward declining age-specific disability levels (Manton and Gu, 2001). Calculating the exact counterfactual (i.e. how much long-term care expenditures would have increased if those functional ability improvements had not occurred) is challenging. Table 3 shows that among persons reaching age 65, the rate of increase in life expectancy has slowed gradually, with a larger increase in the 1970 s of 1.5 years compared to a 0.4 year increase from 1990 to 1997 (roughly the period covered by this study). Manton and Gu (2001) showed that the proportion of the non-institutionalised elderly population with 5 or 6 ADL 
limitations decreased from 3 per cent of the population to 2.9 per cent of the population from 1994 to 1999, and that the rate of people with no disabilities increased about 0.4 per cent per year during this period. Yet a study using the 1968 and 1993 National Mortality Followback Surveys found that disability declines during this period did not affect patterns of nursing home use (Spillman and Lubitz, 2002).

The central issue may hinge on whether compression or expansion of morbidity occurs regardless of the changes in longevity. Goldman and colleagues (2005) calculate three scenarios for future health care expenditures for older people in the US based on different assumptions about future health trends. Their preferred scenario assumes that health status and functional ability decline for future cohorts of older people, based on evidence from the National Health Interview Survey about the current health of young cohorts including increasing rates of obesity and diabetes. The other scenarios assume (1) stable health for entering cohorts and (2) continuing declines in disability at the rates documented by Manton and Gu (2001). The three scenarios yield widely different predictions of spending per beneficiary over the next 30 years.

Based on the results in this study, the most effective policies to constrain future longterm care expenditure growth may be policies that help reduce the likelihood of older people having declines in functional ability beyond three ADL impairments. The finding of substantial increases in long-term care expenditures for increases in ADL impairments beyond three should be interpreted with caution, however, given results of two recent studies. First, Spillman (2004) analysed data from the National Long-Term Care Survey from 1984 to1999 to determine the changes in the distribution of disability over the period. She found that most improvements during this period were among persons with relatively less severe disability, and that the prevalence of persons receiving help with ADLs fell much less than the prevalence of persons receiving help only with IADLs. Therefore, the proportion of people with three or more ADLs was relatively stable. In a different study, Chernew et al. (2005) confirmed that having three or more ADLs was highly predictive of greater health care spending, but also found that growth in expenditures has been greatest among the least disabled persons. These authors concluded that health expenditures are likely to increase inexorably even with substantial declines in severe disability (although their results could change if the rate of cost increases for persons with moderate or low disabilities decreases in the future). Concerns about increasing obesity and associated diseases in the United States and other countries are therefore well-placed. The recent improvements in functional ability could be reversed. While obesity trends could slow or reverse the increases in longevity, they would also undoubtedly impose great costs in terms of increased long-term care expenditures associated with worsened functional status.

The possibility of substantial increases in long-term care expenditures over the next several decades is further supported by work done as part of a recent study in the UK on funding of social care for older people (Wanless, 2006). In order to estimate how trends over the next 20 years are likely to affect the demand for social care, Jagger et al. (2006) developed a simulation model to predict how a set of specific diseases (stroke, coronary heart disease, cognitive impairment/dementia, and arthritis) would affect disability trends for persons over age 65 . Their model showed that ageing of the population by itself (i.e. with no changes in the age-specific prevalence of disease) would result in a 67 per cent increase in the number of persons with disability, and the authors concluded that further increases in longevity would simply result in more years with disability and likely 
greater concomitant health and social expenditures. The predictions in the UK, however, combined with the results from the previous sections would seem to be more optimistic about potential benefits from interventions to reduce disability severity than the work by Chernew and colleagues (2005) discussed above.

One important caveat about our results is that fairly simple models were estimated to focus on the competing roles of increasing age and functional impairment in increasing long-term care expenditures. More sophisticated models incorporating additional variables could modify the level of the estimated marginal effects somewhat. Also, the estimation strategy focused on total ADL impairments rather than a determination of which ADL impairments are most central in explaining long-term care expenditures.

Yet the main point remains: increasing longevity per se is likely to be much less pivotal in increasing future long-term care expenditures than are trends in disabilities and functional status. Age plays a minor role in predicting long-term care expenditures after controlling for disability and proximity to death.

\section{Acknowledgement}

Funding for this research was provided by the Agency for Health Care Policy and Research and the National Institute of Aging (R01-HS09515) and the Demography and Economics of Aging Research Program at the University of North Carolina under grant from the National Institute of Aging (K07 AG01015 and P30 AG04001). The authors are responsible for all analyses and interpretations.

\section{Note}

1 Full regression results are available upon request.

\section{References}

Adler, G.S. (1994), 'A profile of the Medicare current beneficiary survey', Health Care Financing Review, 15(4): 153-163.

Ai, C. \& Norton, E.C. (2000), 'Standard errors for the retransformation problem with heteroskedasticity', Journal of Health Economics, 19(5): 697-718.

Bebbington, A. and Comas-Herrera, A. (2000), Healthy Life Expectancy: Trends to 1998, and the Implications For Long Term Care Costs, PSSRU discussion paper 1695, www.ukc.ac.uk/PSSRU, accessed 11 September 2006.

Bissett, B. (2002), 'Healthy life expectancy in England at subnational level', Health Statistics Quarterly, 14: 21-29.

Bone, M., Bebbington, A., Jagger, C., Morgan, K. and Nicolaas, G. (1995), Health Expectancy and Its Uses, London: HMSO.

Bone, M., Bebbington, A. and Nicolaas, G. (1998), 'Policy applications of health expectancy', Journal of Aging and Health, 10(2): 136-153.

Cambois, E. and Robine, J. (1996), 'An international comparison of trends in disability free life expectancy', in R. Eisen and F. Sloan (eds), Long-term Care: Economic Issues and Policy Solutions, Dordrecht: Kluwer.

Chernew, M.E., Goldman, D.P., Pan, F. and Shang, B. (2005) 'Disability and health care spending among Medicare beneficiaries', Health Affairs 24(6): W5R42-W5R52, Nov.-Dec. 
Cutler, D.M. and Sheiner, L. (1998), 'Demographic and medical care spending: standard and non-standard effects', National Bureau of Economic Research Working Paper Series, Working Paper 6866.

Cutler, D.M. and Meara, E. (2001a), 'Changes in the age distribution of mortality over the 20th century', National Bureau of Economic Research Working Paper Series, Working Paper 8556.

Cutler, D.M. and Meara, E. (2001b), 'The concentration of medical spending: an update', in D.A. Wise (ed.), Themes in the Economics of Aging, Cambridge, MA: The University of Chicago Press, National Bureau of Economic Research.

Diehr, P., Yanez, D., Ash, A., Hornbrook, M. and Lin, D.Y. (1999), 'Methods for analyzing health care utilization and costs', Annual Review of Public Health, 20: 125-144.

Duan, N. (1983), 'Smearing estimate - a nonparametric retransformation method', Journal of the American Statistical Association, 78(383): 605-610.

Efron, B. and Tibshirani, R. (1986), 'Bootstrap measures for standard errors, confidence intervals, and other measures of statistical accuracy', Statistical Science, 1(1): 54-57.

Fries, B. (1980), 'Measuring and monitoring success in compressing morbidity', Annuals of Internal Medicine, 5: 455-459.

Goldman, D.P. et al. (2005), 'Consequences of health trends and medical innovation for the future elderly', Health Affairs, 24(6): W5R5-W5R17, Nov.-Dec..

Jagger, C., Matthews, R., Spiers, N, Brayne, C., Comas-Herrara, A., Robinson, T., Lindesay, J. and Croft, P. (2006), 'Compression or expansion of disability? Forecasting future disability levels under changing patterns of diseases', Nuffield Research Unit, University of Leicester, http://www.kingsfund.org.uk/ resources/publications/appendices_to.html, 7 September 2006.

Little, R.J. (2004), 'To model or not to model? Competing modes of inference for finite population sampling', Journal of The American Statistical Association, 99(466): 546-556.

Manning, W.G. and Mullahy, J. (2001), 'Estimating log models: to transform or not to transform?', Journal of Health Economics, 20(): 461-494.

Manton, K. and Gu, X. (2001), 'Changes in the prevalence of chronic disability in the United States black and nonblack population above age 65 from 1982 to 1999', Proceedings of the National Academy of Sciences, 98(11): 6354-6359.

McGrail, K., Green, B., Barer, M., Evans, R. G., Hertzman, C. and Normand, C. (2000), 'Age, costs of acute and long-term care and proximity to death: evidence for 1987-88 and 1994-95 in British Columbia', Age and Ageing, 29(3): 249-253.

Norton, E.C. (2000), 'Long-term care', in A.J. Culyer and J.P. Newhouse (eds), Handbook of Health Economics, Volume 1B, Elsevier Science B.V.

OECD (1998), Maintaining Prosperity in an Ageing Society, Paris: Organisation for Economic Co-operation and Development.

Olshansky, S.J. and Ault, A.B. (1986), 'The 4th state of the epidemiologic transition - the age of delayed degenerative diseases', Milbank Quarterly, 64(3): 355-391.

Robine, J., Romieu, I. and Cambois, E. (1997), 'Health expectancies and current research', Reviews in Clinical Gerontology, 7: 73-81.

Seshamani, M. and Gray, A. (2004), 'Ageing and health care expenditures: the red herring argument revisited', Health Economics, 13(4): 303-314.

Spillman, B.C. (2004), 'Changes in elderly disability rates and the implications for health care utilization and cost', Milbank Quarterly, 82(1): 157-194.

Spillman, B.C. and Lubitz, J. (2000), 'The effect of longevity on spending for acute and long-term care', New England Journal of Medicine, 342(19): 1409-1415.

Spillman, B.C. and Lubitz, J. (2002), 'New estimates of lifetime nursing home use - have patterns of use changed?', Medical Care, 40(10): 965-975.

Stearns, S.C. and Norton, E.C. (2004), 'Time to include time to death? The future of health care expenditure predictions', Health Economics, 13(4): 315-327.

Van Houtven, C.H. and Norton, E.C. (2004), 'Informal care and health care use of older adults', Journal of Health Economics, 23(6): 1159-1180. 
Sally C. Stearns et al.

Wanless, D. (2006), Securing Good Care for Older People: Taking a Long Term View, King's Fund, http:// www.kingsfund.org.uk/resources/publications/securing_good.html, 7 September 2006.

Yang, Z., Norton, E.C. and Stearns, S.C. (2003), 'Longevity and health care expenditures: the real reasons older people spend more', Journal of Gerontology Series B: Psychological Sciences and Social Sciences, 58B: S2-S10.

Zweifel, P., Felder, S. and Meiers, M. (1999), Ageing of population and health care expenditure: a red herring? Health Economics, 8(6): 485-496. 